\title{
Ações e impactos na redução de óbitos fetais: Percepção de integrantes do comitê de
}

\section{mortalidade}

\author{
Actions and impacts in reducing fetal deaths: Perception of members of the mortality committee \\ Acciones e impactos en la reducción de muertes fetales: Percepción de los miembros del comité de \\ mortalidad
}

Recebido: 14/02/2021 | Revisado: 23/02/2021 | Aceito: 25/02/2021 | Publicado: 04/03/2021

\author{
Maraisa Manorov \\ ORCID: https://orcid.org/0000-0002-0620-4659 \\ Prefeitura Municipal de Araucária, Brasil \\ E-mail: mara_manorov@hotmail.com \\ Alessandra Paiz \\ ORCID: https://orcid.org/0000-0002-4790-0132 \\ Universidade Federal do Paraná, Brasil \\ E-mail: alessandrapaiz@hotmail.com \\ Jeane Barros de Souza \\ ORCID: https://orcid.org/0000-0002-0512-9765 \\ Universidade Federal da Fronteira Sul, Brasil \\ E-mail: jeanebarros18@gmail.com \\ Laura Christina Macedo \\ ORCID: https://orcid.org/0000-0002-1916-2582 \\ Universidade Federal do Paraná, Brasil \\ E-mail: 1.christina.macedo@gmail.com
}

\begin{abstract}
Resumo
Objetivo: desvelar as ações desenvolvidas nos serviços de saúde e seus impactos na redução de óbitos fetais, na percepção de integrantes de um Comitê de Mortalidade do Sul do Brasil. Método: estudo descritivo, de abordagem qualitativa, envolvendo sete profissionais de saúde, membros do Comitê de Mortalidade de um município do Paraná. Realizou-se entrevistas semi-estruturadas, que foram transcritas e analisadas de acordo com a análise de conteúdo. Resultados: Identificaram-se como principais causas para prevenção de óbitos fetais as infecções vaginais e/ou urinárias e entre as dificuldades enfrentadas surgiram a falta de qualificação e rotatividade de profissionais, bem como a falta de adesão ao pré-natal. Para tanto, estabeleceram-se diversas estratégias: elaboração de protocolos, capacitação de profissionais, aquisição de testes, medicamento e monitoramento de prontuários da maternidade. Conclusão: a identificação das fragilidades e posterior implementação de ações contribuiu para redução dos óbitos fetais no município, sendo que o maior impacto envolveu a qualificação dos profissionais para a assistência ao pré-natal.
\end{abstract}

Palavras-chave: Morte fetal; Gravidez; Protocolos; Capacitação profissional; Cuidado pré-natal.

\begin{abstract}
Objective: to unveil the actions developed in health services and their impacts on the reduction of fetal deaths, in the perception of members of a Mortality Committee in the South of Brazil. Method: a descriptive study, with a qualitative approach, involving seven health professionals, members of the Mortality Committee of a municipality in Paraná. Semi-structured interviews were carried out, which were transcribed and analyzed according to the content analysis. Results: Vaginal and/or urinary infections were identified as the main causes for the prevention of fetal deaths. Among the difficulties faced, there was the lack of qualification and turnover of professionals, as well as the lack of adherence to prenatal care. For that, several strategies were established: elaboration of protocols, training of professionals, acquisition of tests, medication and monitoring of the maternity records. Conclusion: the identification of weaknesses and subsequent implementation of actions contributed to the reduction of fetal deaths in the municipality, with the greatest impact involved the qualification of professionals for prenatal care.
\end{abstract}

Keywords: Fetal death; Pregnancy; Protocols; Professional training; Prenatal care.

\section{Resumen}

Objetivo: dar a conocer las acciones desarrolladas en los servicios de salud y sus impactos en la reducción de muertes fetales, en la percepción de los integrantes de un Comité de Mortalidad en el Sur de Brasil. Método: estudio descriptivo, con abordaje cualitativo, que involucró a siete profesionales de la salud, miembros del Comité de Mortalidad de un municipio de Paraná. Se realizaron entrevistas semiestructuradas, que fueron transcritas y analizadas según el análisis de contenido. Resultados: las infecciones vaginales y/o urinarias fueron identificadas como las 
principales causas para la prevención de muertes fetales, entre las dificultades encontradas, la falta de calificación y rotación de profesionales, así como la falta de adherencia a la atención prenatal. Para eso, se establecieron varias estrategias: elaboración de protocolos, capacitación de profesionales, adquisición de pruebas, medicación y seguimiento de los registros de maternidad. Conclusión: la identificación de las debilidades y la posterior implementación de acciones contribuyeron a la reducción de las muertes fetales en el municipio, siendo el mayor impacto la calificación de profesionales para la atención prenatal.

Palabras clave: Muerte fetal; Embarazo; Protocolos; Capacitación profesional; Atención prenatal.

\section{Introdução}

A Taxa de Mortalidade Fetal (TMF) é considerada um dos indicadores mais apropriados para analisar a qualidade da assistência prestada à gestante e ao parto, pois reflete o estado de saúde da mulher, a qualidade, e as condições de acessibilidade e de utilização dos serviços de saúde disponibilizados (Brasil, 2009; Barros, Aquino \& Souza, 2019). Para calcular a TMF são considerados os óbitos fetais ocorridos a partir da $22^{\mathrm{a}}$ semana completa de gestação ou 154 dias, com fetos com peso igual ou superior a $500 \mathrm{~g}$ ou estatura a partir de $25 \mathrm{~cm}$ por mil nascimentos totais na população, residente em um determinado espaço geográfico e ano (Brasil, 2009).

Estima-se que ocorram anualmente por volta de 2,6 milhões de óbitos fetais no mundo, entre os quais $98 \%$ acontecem em países de baixa e média renda. Acredita-se que a maior parte desses óbitos seja provocada por causas preveníveis (Bernis et al., 2016). As principais falhas que contribuem para as altas taxas de mortalidade fetal relacionam-se à qualidade da atenção pré-natal, diagnóstico de alterações na gravidez e manejo obstétrico. Dessa forma, os distintos problemas que conduzem ao desfecho negativo da gestação são relacionados a falhas na capacidade de prevenção e resposta em face de intercorrências durante a gestação e parto (Brasil, 2012).

A evitabilidade dos óbitos fetais é reconhecida como indicador da efetividade da assistência à saúde, podendo direcionar a disponibilidade de recursos necessários para o planejamento de ações, objetivando a melhoria da assistência prénatal e ao parto (Barros, Aquino \& Souza, 2019). Algumas ações básicas possibilitam promover uma resposta mais positiva sobre a sobrevida e redução da TMF, tais como: qualificação do cuidado; a ampliação do acesso aos serviços, desde o pré-natal à assistência hospitalar ao parto e ao nascimento; pronto reconhecimento de situações de risco; e a provisão do cuidado apropriado e resolutivo à gestante (Brasil, 2009).

Para tanto, conhecer a epidemiologia da morte fetal e identificar os fatores determinantes dos óbitos é fundamental para subsidiar a adoção de medidas preventivas e promover ações voltadas à saúde materno-infantil, o que permite o enfrentamento mais efetivo desse problema evitável (Menezzi et al., 2016).

No Brasil a vigilância do óbito infantil e fetal foi instituída por meio de base legal e é obrigatória nos serviços de saúde que integram o Sistema Único de Saúde (SUS), (Brasil, 2010), sendo executada pelos Comitês de Prevenção do Óbito Infantil e Fetal. Os Comitês possuem caráter educativo, formativo, com as atribuições de dar visibilidade, acompanhar, monitorar os óbitos infantis e fetais e propor intervenções para redução da mortalidade (Brasil, 2009). Neste cenário, despontou a pergunta de pesquisa: quais as ações desenvolvidas nos serviços de saúde e seus impactos na redução dos óbitos fetais, na percepção de integrantes de um Comitê de Mortalidade do Sul do Brasil?

Nesse contexto, a realização deste estudo se justifica ao considerar a importância da atuação dos Comitês de Prevenção do Óbito Infantil e Fetal na análise da mortalidade fetal, em busca de evitar a sua ocorrência e auxiliar na organização dos serviços, de modo a planejar ações efetivas para qualificar a assistência prestada à gestante e ao recém-nascido (Barros, Aquino \& Souza, 2019; Brasil, 2009; Ruoff, Schmitt \& Andrade, 2017). Assim, o objetivo do estudo foi desvelar as ações desenvolvidas nos serviços de saúde e seus impactos na redução de óbitos fetais, na percepção de integrantes de um Comitê de Mortalidade do Sul do Brasil. 


\section{Metodologia}

Trata-se de um estudo descritivo de abordagem qualitativa, em que a interpretação do pesquisador e suas opiniões sobre o fenômeno de estudo tornam-se importantes e a coleta de dados é realizada, geralmente, por meio de entrevistas com questões abertas (Pereira, Shitsuka, Parreira \& Shitsuka, 2018).

O estudo foi desenvolvido com os integrantes do Comitê de Mortalidade de um município da região metropolitana de Curitiba, Paraná, Brasil. Contou-se com a participação de sete profissionais de saúde que atuavam no referido Comitê. Considerou-se como critérios de inclusão: atuar há mais de seis meses como membro do Comitê de Mortalidade do município.

Para identificar e contatar os profissionais contou-se com o apoio da coordenadora da vigilância epidemiológica do município, que disponibilizou relação com o nome dos participantes do Comitê de Mortalidade e uma data de reunião do grupo para que fosse realizado convite para participação da pesquisa. Todos os participantes do grupo foram convidados, no total sete profissionais de saúde, não havendo recusas.

Inicialmente, a pesquisa foi apresentada aos integrantes do Comitê e após realizou-se o convite para participarem, assinando o Termo de Consentimento Livre e Esclarecido (TCLE). A coleta de dados ocorreu no primeiro semestre de 2020, em entrevista semiestruturada, pré-agendada e presencial, na sala de reuniões da sede da Secretaria de Saúde do município, com duração média de vinte minutos. Para tanto, utilizou-se um roteiro de questões para nortear a entrevista, as quais foram gravadas em smartphone e transcritas na íntegra. Respeitou-se o anonimato dos participantes utilizando-se de siglas para identificar as falas, com a abreviação da palavra entrevistado (E) seguidas de um número.

A análise de dados foi realizada a partir das transcrições das entrevistas, em que se utilizou o método de análise de conteúdo (Bardin, 2011). Primeiramente realizou-se a pré-análise com leitura flutuante dos dados extraídos nas entrevistas, construção de tabela com os dados coletados e escolha dos documentos para a constituição dos dados para os procedimentos analíticos. Em seguida, explorou-se o material de análise, organizando-os em duas categorias: 1) Dificuldades encontradas; 2) Efetividade das ações e seus resultados. Este estudo foi aprovado em 13 de abril de 2020, pelo Comitê de Ética em Pesquisa com Seres Humanos de uma Universidade Pública do Sul do Brasil, sob o parecer número 3.966.324.

\section{Resultados e Discussão}

Participaram do estudo sete profissionais da saúde, com idade entre 29 e 40 anos, seis do sexo feminino e um do sexo masculino, entre eles quatro enfermeiros, uma nutricionista e duas médicas, sendo uma ginecologista e a outra pediatra. Quanto ao tempo de atuação no município, este variou de um a doze anos e como integrantes do comitê de mortalidade entre um a dois anos.

\section{Dificuldades encontradas}

Compreender e identificar as principais causas e fatores de risco associados aos óbitos fetais é essencial para avaliar o risco de recorrência e possibilitar o planejamento de estratégias de prevenção e consequentemente reduzir a taxa de mortalidade fetal (Serrano, Centeno \& Ramalho, 2018).

Ficou evidente que no primeiro semestre de 2019, o município de estudo contava com um alto número de óbitos fetais. Diante disso, os integrantes do Comitê de Mortalidade decidiram investigar e discutir as causas para estabelecer melhores estratégias, em busca de controlar a situação:

O primeiro semestre de 2019 fechou com 29 óbitos infantil e fetal. (E1)

Desde o momento que a gente identificou as falhas, começamos a estudar o que estava acontecendo e conseguimos fazer o levantamento de problemas, identificamos atores que estavam envolvidos. (E2) 
Entre as principais causas de óbitos fetais identificaram-se as infecções no período perinatal (infecções do trato urinário e vaginoses):

“A gente teve no começo do ano passado [2019], acho que foram quatro óbitos por vaginose/vaginite e mais três por infecção do trato urinário não tratado”. (E1)

As infecções durante o período gestacional são um fator de risco para mortalidade fetal. Para tanto, desvela-se a importância da assistência pré-natal que pode contribuir para desfechos favoráveis, pois permite a detecção precoce e o tratamento de infecções. Somado a isso, controla os fatores de risco que podem trazer complicações para a saúde da mãe e do feto (Moura, Alencar, Silva \& Almeida, 2018). Nesse contexto, pode-se inferir que as infecções são causas preveníveis de óbitos fetais e que dependem de uma efetiva assistência ao pré-natal.

A falta de qualificação dos profissionais da Atenção Primária à Saúde (APS) para a assistência ao pré-natal foi mencionada pelos participantes como uma dificuldade enfrentada:

A gente vê o mesmo profissional cometendo o mesmo erro duas, três vezes [...] podia ter evitado [...] colocando um dado a mais no prontuário [...] olhando um exame que não viu. (E3)

Muitas vezes a gente pega os prontuários e vê que poderia ter sido evitado, alguma coisa que poderia ter sido melhorada ou [...] ter deixado de ser feito alguma coisa, por exemplo não pediram tal exame ou não viram corrimento. (E4)

[...] $90 \%$ dos óbitos eram de [...] gestante de baixo risco, onde os profissionais da ponta não sabiam manejar o prénatal. (E1)

A maioria dos óbitos era por condutas inadequadas na atenção a gestante, no pré-natal, em nível de atenção básica. (E5)

Ao longo dos anos houve uma progressiva expansão do processo de organização dos serviços de APS nos municípios, demonstrando um aumento na cobertura da atenção pré-natal no Brasil. Contudo, essa atenção não pode ser considerada adequada, visto que a qualificação dos profissionais de saúde continua sendo um desafio (Brasil, 2013) Entre os fatores que contribuem para a baixa qualidade da assistência estão a não realização de exames laboratoriais de rotina, procedimentos básicos recomendados, prescrições e orientações durante as consultas (Brasil, 2013; Nunes, Gomes, Rodrigues \& Mascarenhas, 2016).

Outro motivo que pode influenciar a qualidade da assistência prestada no pré-natal, citado como uma dificuldade pelos participantes, é a rotatividade de profissionais nos serviços:

A rotatividade de profissional, [...] muda muito. (E3)

A rotatividade de profissionais porque, muitas vezes, a gente faz capacitação, orienta toda a unidade e dois meses depois sai o médico, o enfermeiro, só fica praticamente o ACS. (E6)

A gestação é considerada um momento especial para muitas mulheres. Assim, criam-se expectativas com relação ao atendimento gestacional, esperando estabelecer vínculo de confiança com os profissionais de saúde, o que pode ser prejudicado com a rotatividade dos profissionais e acarretar na má adesão ao pré-natal (Rocha, Barbosa \& Lima, 2017). Essa rotatividade além de implicar na dificuldade dos profissionais estabelecerem vínculo com as mulheres gestantes e suas famílias, compromete a qualidade e a longitudinalidade do cuidado (Tonelli, Leal, Tonelli, Veloso, Gonçalves \& Tonelli, 2018). 
Nesse sentido, a adesão e frequência às consultas no período gestacional podem ser influenciadas pela maneira que essas mulheres são acolhidas, pelo apoio recebido e assistência prestada. Mas existem outros fatores, inerentes às mulheres, que podem interferir negativamente na sua adesão ao cuidado, como possuir tradições familiares de descrença ao pré-natal (Rocha, Barbosa \& Lima, 2017). A falta de adesão ao pré-natal foi referida como uma dificuldade enfrentada:

\section{A adesão ao pré-natal era um grande problema. (E2)}

Falta uma responsabilidade da população, dessa gestante em perceber que o pré-natal é importante. (E1)

A gente pode fazer tudo certinho, se a paciente não fizer o lado dela, não vai ter um resultado bom [...] as vezes, a própria paciente não procurou ajuda antes. (E4)

A adesão ao pré-natal permite o acompanhamento detalhado das condições de saúde da gestante e seu feto, prevenindo e/ou tratando as complicações que podem surgir nesse período, possibilitando a diminuição dos índices de morbimortalidade materna e infantil (Pereira, Ferreira, Araújo, Melo \& Andrade, 2018). Dessa maneira, ao reconhecer as principais causas de óbitos e as dificuldades relacionadas a assistência pré-natal, os membros do Comitê de Mortalidade estabeleceram estratégias, com vistas a fortalecer e qualificar a atenção prestada às gestantes, em busca de reduzir a mortalidade fetal do município.

\section{Efetividades das ações e seus resultados}

A mortalidade fetal é um impactante fator para a saúde pública, por se tratar de um indicador de desenvolvimento de saúde em determinadas regiões do país e da qualidade da assistência prestada a gestante e ao parto (Serrano, Centeno \& Ramalho, 2018). Portanto, para reduzir a TMF no Brasil é necessário investir nos comitês de investigação de óbitos fetais auxiliando no planejamento de ações para consolidar a rede assistencial perinatal, reestruturando e qualificando os cuidados pré-natais (Barros, Aquino \& Souza, 2019; Silva et al., 2019).

Nesse sentido, o município do estudo investiu os recursos necessários no Comitê de Mortalidade, possibilitando aos integrantes realizarem diversas pesquisas, baseando-se na literatura científica, a fim de aprimorar suas ações. A partir de então, implementaram diversos elementos nos serviços de saúde, em prol da redução da mortalidade fetal, conforme demonstração no Quadro 1: 
Quadro 1 - Ações para redução de óbitos fetais.

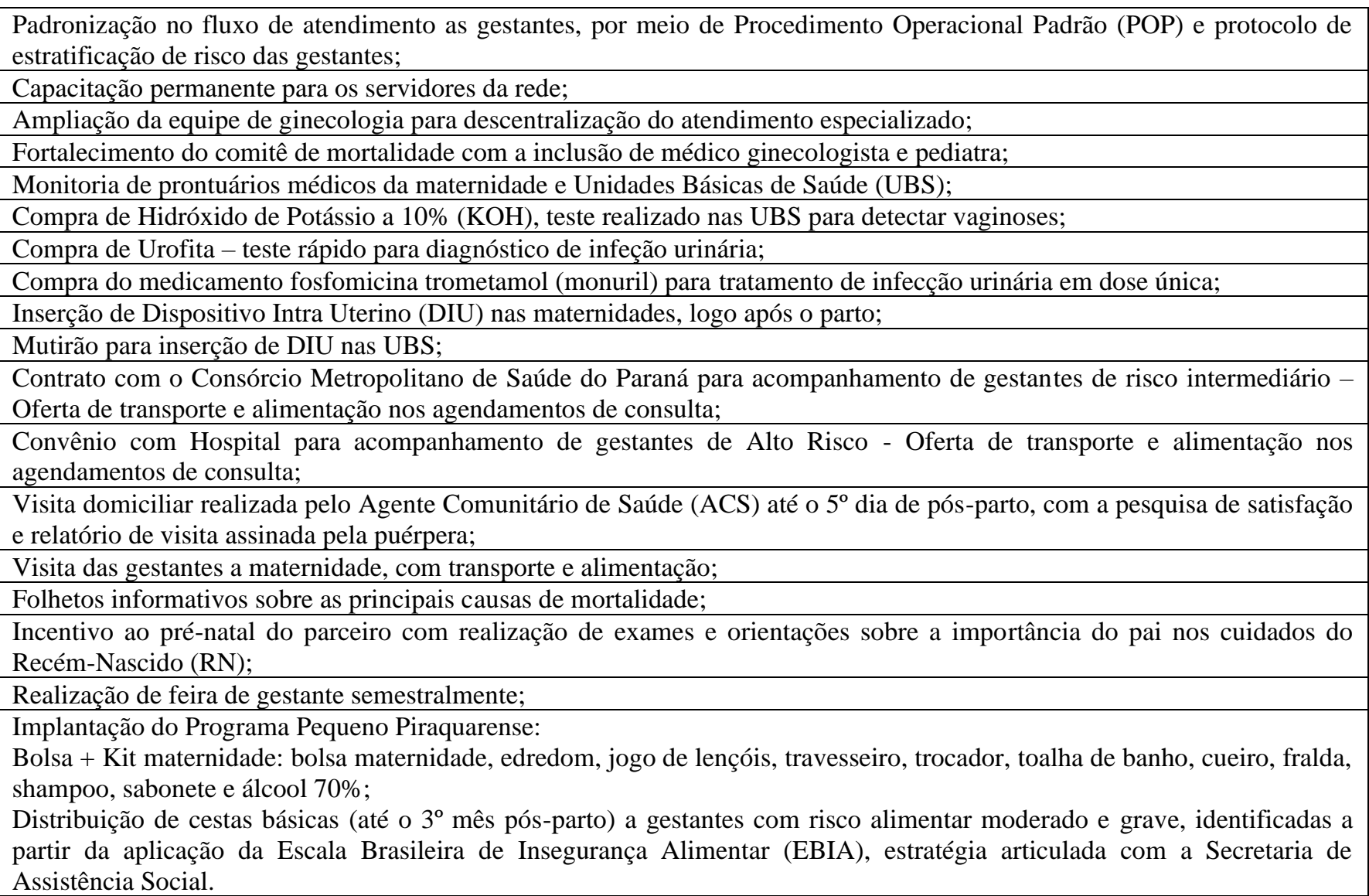

Fonte: Autoras (2020).

Os participantes do estudo referiram que uma das ações que consideraram mais efetivas foi a elaboração do protocolo de atenção às gestantes:

Elaboração dos protocolos, a padronização das unidades de saúde do atendimento na primeira consulta e a todo o pré-natal. (E2)

Novos protocolos que foram instituídos, de o que não pode faltar numa anamnese, exame físico, o que não pode deixar de ser visto. (E4)

O protocolo de pré-natal, embasado nas recomendações do Ministério da Saúde (MS), estabeleceu o número mínimo de oito consultas, com acompanhamento intercalado entre médicos e enfermeiros, priorizando o início do pré-natal até a $12^{a}$ semana. Estipulou exames a serem solicitados em cada trimestre da gestação, incluindo o teste de aminas para diagnosticar infecções vaginais, com indicação de medicamentos para o tratamento dessas infecções e das urinárias, bem como de outras queixas. Determinou a estratificação do risco gestacional, conforme a proposta da Secretaria de Saúde do Paraná, definindo fluxos para encaminhamento das gestantes para avaliação e/ou seguimento aos serviços de maior complexidade, salientando a continuidade do acompanhamento na APS. Além de orientações referentes às principais alterações durante a gravidez e importância da amamentação.

Para a implementação dos protocolos, tornou-se necessária a capacitação dos profissionais da rede de atenção do município: 
A capacitação com a equipe foi fundamental. (E5)

Mudança na consciência das pessoas que trabalham no município [...] com as capacitações que foram feitas [...] as pessoas da secretaria prepararam bem os profissionais para dar as orientações. (E4)

Com tanta capacitação que a gente faz, batendo na tecla, hoje eu vejo que os profissionais conseguem manejar e quando não conseguem, se preocupam, que é também o que a gente queria, se não sabe fazer isso, pelo menos se preocupa e vai atrás da informação. (E1)

A elaboração do protocolo e a capacitação dos profissionais da APS contribuíram para reciclar e construir novos conhecimentos, servindo como ferramenta para aperfeiçoar a assistência ao pré-natal (Ribeiro, Souza \& Silva, 2019) e minimizar a dificuldade da falta de qualificação profissional.

Outra estratégia apontada pelos participantes foi a aquisição de testes e medicamentos pela gestão municipal e disponibilização nas UBS e maternidade, pois contribuiu para a eficácia no atendimento, auxiliando na detecção de infecções vaginais e urinárias, tratamento em tempo oportuno e consequente redução de óbitos por essas causas:

\section{Implementação da utilização do KOH. (E2)}

Colocar o KOH foi essencial, a mulher já sai com o resultado e a medicação pronta para ir para casa. (E1)

Implementação da utilização da urofita. (E2)

Colocar o monuril foi essencial. (E1)

Eles conseguiram o monuril, para tomar em dose única que, muitas vezes, a gestante não conseguia tomar os sete dias de antibiótico e isso prejudicava bastante. (E6)

O Hidróxido de potássio a $10 \%(\mathrm{KOH})$ foi adquirido com o objetivo de diagnosticar vaginose bacteriana em

gestantes. É considerado um método eficaz e de baixo custo, sugerido como método de rotina nas consultas de pré-natal (Campos et al., 2012). Portanto, passou a fazer parte do protocolo de exames realizados, obrigatoriamente para mulheres com idade gestacional entre 24 e 28 semanas ou em qualquer período para quem manifestasse sintomas.

O teste de urina (Urofita) foi implementado para tornar o diagnóstico rápido e oportunizar o tratamento precoce de infecções urinárias, que são eventos frequentes na gestação, devido às modificações anatômicas no corpo da mulher somadas as alterações emocionais (Calixto et al., 2019). Para facilitar o tratamento dessas infecções foi obtida a fosfomicina trometamol (monuril), administrado em dose única, diminuindo custos, melhorando a adesão e tolerância das usuárias ao tratamento (Calixto et al., 2019), a qual foi disponibilizada para as farmácias das UBS e na maternidade de referencia do município.

Somado a estas ações, o monitoramento dos prontuários da maternidade foi apontado por todos os participantes como a ação mais importante realizada:

O monitoramento dos prontuários da maternidade foi o melhor pra redução de mortalidade. (E3)

O monitoramento da maternidade [...] a ação mais importante que a gente desenvolveu. (E2)

O município em estudo não tem maternidade "em seu território", assim as gestantes são atendidas em outra cidade e não é realizada a contra referência para as UBS. Portanto, o monitoramento diário dos prontuários da maternidade permitiu que os profissionais da APS soubessem quem eram as gestantes que buscaram atendimento, para adotar condutas necessárias: 
Como a gente não tem maternidade no município não é interligada as equipes, quando fazemos o monitoramento conseguimos saber o que aconteceu, porque essa gestante foi lá [na maternidade]. Eram essas gestantes que iam lá e que a gente não sabia que tinham ido, que complicavam em casa e acaba culminando com óbito (E7)

A gente tem uma senha do sistema da maternidade e todo dia [...] tem um profissional que acessa esse sistema, olha quais são as gestantes que passaram pelo pronto atendimento da maternidade, com quais queixas e se está internada ou não, se precisa de busca ou não, e com isso a gente localiza de qual unidade é, quem é o agente comunitário de saúde, e manda um memorando pra unidade de saúde fazer busca se precisar e ir atrás dessa gestante para orientar ou agendar consulta. (E3)

Vale ressaltar que a falha na contra referência é apontada como fator que dificulta o processo de assistência dos profissionais da APS (Fenandez et al., 2016), o que influencia na qualidade da atenção prestada, bem como na longitudinalidade do cuidado às gestantes. Ao perceber essa carência e necessidade, os integrantes do Comitê de Mortalidade encontraram uma alternativa para suprir essa falha, realizando o monitoramento diário dos prontuários da maternidade. Dessa forma, as gestantes que necessitavam de atenção diferenciada, devido a complicações, passaram a ser acolhidas pelos profissionais de suas respectivas UBS, as quais buscam auxiliar na resolução de suas demandas. Fator que contribui para o estabelecimento e fortalecimento de vínculo, permitindo uma relação de confiança entre a equipe de saúde e gestante (Souza et al., 2019), melhorando a adesão ao pré-natal.

O fato é que logo após a implementação das ações nos serviços de saúde, os resultados positivos já começaram a despontar:

Se a gente for comparar o primeiro semestre com o segundo eu entendo que os resultados foram bem positivos, até maiores do que a gente esperava. (E7)

Tivemos resultados positivos e, por incrível que pareça, mais rápido do que a gente imaginou. (E3)

Foram bem significativos [...], eficazes, tanto que a gente manteve a maioria das ações. (E2)

A gente foi de uma taxa de 55, que estava em janeiro do ano passado [...] e finalizou o ano com uma taxa de 16. (E1)

Em número absoluto de óbitos a gente teve uma redução de 60-65\%. (E7)

A implementação de todas as ações, em conjunto com os esforços dos membros do Comitê de Mortalidade e de todos os profissionais da rede de atenção, possibilitou que resultados positivos fossem alcançados, qualificou-se o processo de cuidados das gestantes e, consequentemente, os números de óbitos fetais foram reduzidos no município. Entretanto, nenhuma ação foi estabelecida com o intuito de diminuir a rotatividade dos profissionais no município, visto que esse é um fator fundamental no que tange a manutenção da qualidade da assistência ao pré-natal (Martins, Silva, Matos \& Salum, 2019). Nesse sentido, pode-se inferir que depois de capacitados os profissionais para uma atenção efetiva às gestantes e consequente redução de óbitos fetais, muitos desses profissionais qualificados deixam o município e novamente o ciclo se inicia, com o aumento do número de óbitos. Para tanto, cabe a reflexão de que é premente que a gestão municipal elabore estratégias para manter os profissionais capacitados no município e dessa forma consiga reduzir ainda mais os números de óbito materno e fetal. 


\section{Conclusão}

$\mathrm{O}$ alto índice de mortalidade fetal instigou os integrantes do Comitê de Mortalidade do município a investigarem as principais causas de óbitos fetais e as dificuldades relacionadas à assistência prestada às gestantes durante o acompanhamento pré-natal. Identificaram-se como principais causas para prevenção de óbitos fetais as infecções vaginais e/ou urinárias e entre as dificuldades enfrentadas surgiram a falta de qualificação e rotatividade de profissionais, bem como a falta de adesão ao prénatal. Diante dessas necessidades e com vistas a qualificar a atenção pré-natal e reduzir a mortalidade fetal, foram estabelecidas diversas estratégias.

As ações mais eficazes se relacionaram, principalmente, com a qualificação dos profissionais para a assistência ao pré-natal, tais como: elaboração de protocolo; capacitação dos profissionais da saúde; aquisição e disponibilização de testes para diagnóstico de infecções urinárias e vaginais; medicação para os tratamentos. Porém, o monitoramento dos prontuários da maternidade foi mencionado como umas das ações instituídas mais efetiva, pois permitiu a longitudinalidade do cuidado às gestantes.

As limitações da pesquisa se referem ao curto espaço de tempo entre a implementação das ações e a realização da pesquisa, bem como a necessidade de isolamento social provocada pelo risco de contágio do COVID-19, o que dificultou a continuidade do estudo.

Sugere-se a realização de outros estudos com o propósito de identificar as ações realizadas pelos municípios para a redução da mortalidade fetal/materna/infantil, considerando a importância de divulgá-las, visto que esse problema é uma realidade, tanto no Brasil como em diversos países, principalmente nos de baixa e média renda, com o intuito de multiplicar as ações efetivas no território nacional.

\section{Referências}

Bardin L (2011). Análise de Conteúdo: Edições 70, LDA.

Barros, P. S., Aquino, E. C., \& Souza, M. R. (2019, janeiro 31). Mortalidade fetal e os desafios para a atenção à saúde da mulher no Brasil. Revista de Saúde Pública, 53(12). https://doi.org/10.11606/s1518-8787.2019053000714

Bernis, L., Kinney, M. V., Stones W., Hoope-Bender, P., Vivio, D., Leisher, S. H., Bhutta, Z. A., Gülmezoglu, M., Mathai, M., Belizán, J. M., Franco, L., McDougall, L., Zeitlin, J., Malata, A., Kim, E., \& Dickson, J. E. L. (2016). Stillbirths: ending preventable deaths by 2030. Lancet, 387 (10019), $703-16$. PubMed PMID: 26794079. http://dx.doi.org/10.1016/S0140-6736(15)00954-X

Calixto, A. C. V., Meleipe, J. G. G., Bacelar, M. C. P., Gonçalves, P. G., Jardim, P. H. M., \& Esteves, A. P. V. S. (2019). Infecção urinária na gravidez. Revista Caderno de Medicina, 2(1). http://www.revista.unifeso.edu.br/index.php/cadernosdemedicinaunifeso/article/download/1289/574

Campos, A. A. S., Leite, A. P. L., Lisboa, C. V. F., Andrade, C. C., Bezerra, A. F., Mattar, R., \& Souza, E. (2012). Estudo comparativo entre o teste do pH e do $\mathrm{KOH}$ versus escore de Nugent para diagnóstico da vaginose bacteriana em gestantes. Rev. Bras. Ginecol. Obstet., 34(5): 209-14. https://doi.org/10.1590/S0100-72032012000500004

Fernández, M. A., Roo, J. P., Irigoyen, A. C., Blanco, S. L., Edward, A. B., \& Juárez, V. T. (2016). Os Sistemas de Referência e Contra-Referência de pacientes na América Latina: Mecanismos de Coordenação Assistencial e papel da Medicina de Família e Comunidade. Rev Bras Med Fam Comunidade, 11(Suppl 2): 37-45. http://dx.doi.org/10.5712/rbmfc11(0)1384

Martins, M. S., Matos, E., \& Salum, N. C. (2019, February 14). Rotatividade dos trabalhadores de enfermagem em uma unidade de emergência adulto. Texto \& Contexto - Enfermagem, 28(e20160069). https://dx.doi.org/10.1590/1980-265x-tce-2016-0069

Menezzi, A. M. E. D., Figueiredo, I. D., Lima, E. W. B., Almeida, J. C., Marques, F. K. S., Oliveira, C. F., Barreto, N. A. P., \& Pinho, L. (2016). Vigilância do óbito fetal: estudo das principais causas. O Mundo da Saúde, São Paulo, $40(2)$ : 208-12. http://bvsms.saude.gov.br/bvs/periodicos/mundo_saude_artigos/vigilancia_obito_fetal.pdf

Ministério da Saúde \& Departamento de Ciência e Tecnologia (2012). Síntese de evidências para políticas de saúde: mortalidade perinatal: Ministério da Saúde. http://bvsms.saude.gov.br/bvs/publicacoes/sintese_evidencias_mortalidade_perinatal.pdf

Ministério da Saúde, Portaria $n^{\circ} 72$, de 11 de janeiro de 2010. Estabelece que a vigilância do óbito infantil e fetal é obrigatória nos serviços de saúde (públicos e privados) que integram o Sistema Único de Saúde (SUS). http://bvsms.saude.gov.br/bvs/saudelegis/gm/2010/prt0072_11_01_2010.html

Ministério da Saúde, Secretaria de Atenção à Saúde \& Departamento de Atenção Básica (2013). Atenção ao pré-natal de baixo risco: (1a ed.) ver. http://bvsms.saude.gov.br/bvs/publicacoes/atencao_pre_natal_baixo_risco.pdf 
Research, Society and Development, v. 10, n. 3, e4610312964, 2021

(CC BY 4.0) | ISSN 2525-3409 | DOI: http://dx.doi.org/10.33448/rsd-v10i3.12964

Ministério da Saúde, Secretaria de Atenção à Saúde, Departamento de Ações Programáticas \& Saúde da Criança e Aleitamento Materno (2009). Manual de vigilância do óbito infantil e fetal e do Comitê de prevenção do óbito infantil e fetal. Brasília: Ministério da Saúde. http://bvsms.saude.gov.br/bvs/publicacoes/manual_obito_infantil_fetal_2ed.pdf

Moura B. L. A., Alencar, G. P., Silva Z. P., \& Almeida M. F. (2018). Internações por complicações obstétricas na gestação e desfechos maternos e perinatais, em uma coorte de gestantes no Sistema Único de Saúde no Município de São Paulo, Brasil. Cad. Saúde Pública, 34(1): e00188016. https://doi.org/10.1590/0102-311x00188016.

Nunes J. T., Gomes K. R. O., Rodrigues M. T. P., \& Mascarenhas M. D. M. (2016). Qualidade da assistência pré-natal no Brasil: revisão de artigos publicados de 2005 a 2015. Cad. Saúde Colet., 24(2): 252-61. Recuperado em 15 agosto, 2020, de https://www.scielo.br/pdf/cadsc/v24n2/1414-462X-cadsc-24-2-252.pdf

Pereira, A. S., Shitsuka, D. M., Parreira, F. J., \& Shitsuka, R. (2018). Metodologia da pesquisa científica. UFSM. https://repositorio.ufsm.br/bitstream/handle/1/15824/Lic_Computacao_Metodologia-Pesquisa-Cientifica.pdf?sequence=1

Pereira, D. O., Ferreira, T. L. S., Araújo, D. V., Melo, K. D. F., \& Andrade, F. B. (2018). Avaliação das consultas de pré-natal: adesão do pré-natal e complicações na saúde materno-infantil. Revista Ciência Plural, 3(3): 2-15. https://periodicos.ufrn.br/rcp/article/view/12891

Ribeiro, B. C. O., Souza, R. G., \& Silva, R. M. (2019). A importância da educação continuada e educação permanente em unidade de terapia intensiva revisão de literatura. Rev Inic Cient e Ext. 2(3): 167-75. https://revistasfacesa.senaaires.com.br/index.php/iniciacaocientifica/article/view/253/193

Rocha, I. M. S., Barbosa, V. S. S., \& Lima, A. L. S. (2017). Fatores que influenciam a não adesão ao programa de pré-natal. Revista Recien, 7(21): 21-9. https://doi.org/10.24276/rrecien2358-3088.2017.7.21.21-29

Ruoff, A. B., Andrade S. R., \& Schmitt M. D. (2017, Mar 7). Atividades desenvolvidas pelos comitês de prevenção do óbito infantil e fetal: revisão integrativa. Rev Gaúcha Enferm, 38(1): e67342. http://dx.doi. org/10.1590/1983-1447.2017.01.67342

Serrano, F., Centeno, M., \& Ramalho, C. (2018). Estudo das situações de morte fetal após as 24 semanas. Acta Obstétrica e Ginecológica Portuguesa, 12(3), 240-244. http://www.scielo.mec.pt/scielo.php?script=sci_arttext\&pid=S1646-58302018000300015\&lng=pt\&tlng=p

Silva, V. M. C., Tavares, N. H. F., Silva, M. B., Silva, I. C., Rêgo, T. C., Silva, D. F. S., Silva, T. R. S., Dias, M. C. J., Barros, K. V. M., Silva, A. C. F. A., Andreto, L. M., Silva, E. V., Sales, C. C. S., Almeida, S. T., \& Machado, S. P. C. (2019). Fatores associados ao óbito fetal na gestação de alto risco: Assistência de enfermagem no pré-natal. REAS/EJCH, 37(e1884). https://doi.org/10.25248/reas.e1884.2019

Souza, M. C. T., Viana, M. M. L., Leite, D. P., Silva, M. K. N., Bessa, F. C., Bento, D. A. B., \& Santos, R. L. S. (2019). A Importância do Vínculo Profissional Atribuída por Gestante. Rev.Mult. Psic., 13(46), 938-45. https://idonline.emnuvens.com.br/id/article/view/1941

Tonelli, B. Q., Leal, A. P. R., Tonelli, W. F. Q., Veloso, D. C. M. D., Gonçalves, D. P., \& Tonelli, S. Q. (2018, May/Aug). Rotatividade de profissionais da Estratégia Saúde da Família no município de Montes Claros, Minas Gerais, Brasil. RFO UPF, 23(2): 180-85. http://dx.doi.org/10.5335/rfo.v23i2.8314 